\title{
Vaginal cuff closure at total laparoscopic hysterectomy (TLH): Laparoscopic suturing versus vaginal closure
}

\author{
S A Karunananda ${ }^{a}$
}

\begin{abstract}
Total laparoscopic hysterectomy (TLH), is fast replacing the conventional open procedure where and when feasible with greater public awareness and demand. Lesser disastrous major complications and affordability will help towards this trend in making it more popular. Any measures to improve and simplify and speed up this procedure demanding a higher level of skill, should be welcome.

A total number of 62 TLH procedures, performed at the Teaching Hospital Peradeniya were followed up for a minimum of 4 months and analysed to see the speed and complications related to the method of vaginal cuff closure. Twenty six (42\%) TLH procedures were done entirely trans abdominally with the laparoscope (A), and the rest $36(58) \%$ had the cuff closed manually, through vaginal approach (V).

Complications directly attributable to surgery on the closure of the vaginal cuff itself were much higher with the abdominal technique-6 (23\%) instances; but only 3 (8.3\%) in the vaginal approach.

The average vaginal closure time was over 3 times faster vaginally (19 vs 5.5min) with a lesser complication rate,making it the personal preference of the author as the research progressed explaining the higher proportion of vaginal closures.
\end{abstract}

Key words: vaginal vault closure laparoscopic hysterectomy

Sri Lanka Journal of Obstetrics and Gynaecology 2021; 43: 59-62

DOI: http://doi.org/10.4038/sljog.v43i1.7978

a Senior Lecturer in Obstetrics and Gynaecology, Faculty of Medicine, University of Peradeniya, Sri Lanka.

Correspondence: SAK, e-mail: asokakk@hotmaIl.com

DD https://orcid.org/0000-0001-8811-0148

Received $18^{\text {th }}$ February 2021

Accepted after revision $2^{\text {nd }}$ March 2021 


\section{Introduction and objectives}

We rarely give second thoughts to the final step of closing the vaginal vault following the more involving step of removal of the uterus focusing to spare the ureters at TLH procedure. Yet the closure of the vaginal vault is often the most difficult part of TLH due to the difficulty of laparoscopic suturing techniques, more so before advent of barb sutures. Laparoscopic opening of vault invariably causes more ischaemia and heat damage to vaginal edge which is often dissected to a lesser extent than with open surgery. Therefore vaginal closure needs more attention at TLH as suboptimal outcome and complications are often related to this step.

Total laparoscopic hysterectomy was performed in only under $15 \%$ of total number of hysterectomies at the Teaching Hospital Peradeniya for a multitude of reasons. The author who has been performing cuff closure abdominally since 1998, first resorted to performing cuff closure following TLH vaginally, when the only laparoscopic needle holder available at the time failed. It was since felt to be a blessing in disguise when the relative ease and speed of vaginal closure was realized, encouraging more frequent adaptation. The technique was most convenient before the advent of barb sutures making laparoscopic suturing less time consuming. A comparison of the procedures for efficacy and safety is attempted from Jan 2012 to August 2020.

\section{Method}

A retrospective comparative analysis between the abdominal laparoscopic cuff closure (abdominal) (A) against the vaginal closure $(\mathrm{V})$ of the vault of 62 women undergoing TLH at TH Peradeniya from January 2012 to August 2020. Mean age of patients was 44.8 years.

All surgeries were for benign indications; most commonly for intractable dysfunctional uterine bleeding and fibroids under $7 \mathrm{~cm}$ (mostly removed with the morcellator). Most procedures in older patients had adnexae removed (bilateral salpingo-oophorectomy) 2 patients had sacro colpopexy performed simultaneously using mash and a tacking device. Four patients, needing small cystocele repairs had vaginal cuff closure for convenience. The time taken to repair the cystoceles or vault suspensions were not added to cuff closure times.

Majority of the procedures 52 (84\%), were performed by author himself and the rest by a registrar or senior registrar under direct supervision. Though, the author performed similar surgery in the private sector such cases were not included for the study.

All the TLH procedures were performed by traditional laparoscopic hysterectomy with a $10 \mathrm{~mm}$ laparoscope inserted through $12 \mathrm{~mm}$ port and two or three $5 \mathrm{~mm}$ side ports. Most of operations were completed with bipolar coagulation and scissors and sparing use of harmonic scalpel and power blade device when available. Circular colpotomy was performed with the monopolar hook and bipolar scissors or the harmonic scalpel. Uteri were delivered mostly vaginally but morcellated when distended with large fibroids. Intra operative antibiotics and tranexamic acid $500 \mathrm{mg}$ to $1000 \mathrm{mg}$ intra venous were used in nearly every occasion. Larger dozes were used when extra dissections were required due to larger uteri or adhesions.

The total number of cases were 62 of which 26 (42\%) had vault closure laparoscopically and the rest 36 (58)\% vaginally. Continuous Barb sutures - (unidirectional) was used in 18 cases of $\mathrm{A}$ as it seemed to speed up matters though no attempt was made to compare the time advantage within the abdominal closure group (barb Vs conventional sutures) as this was not the main objective of the study. All abdominal cuff closures were sutured intra-corporeal continuous, with no 0 thickness polyglactin 910 or barb sutures. Vaginal cuff was sutured in vaginal approach, with no 1 interrupted Polyglactin 910, usually as a figure of eight suture being placed at the centre of the cuff alongside extra sutures for a broader vault.

In one procedure involving a difficult cervical fibroid, the vaginal cuff was left open with application of a running stitch to the edge, due to oozing, to facilitate drainage. It was sutured the following day, vaginally, after removal of a pressure pack left in the vagina.

The method of cuff closure selected for any given patient was on the merit of findings at surgery and were only occasionally predetermined. Obese patients with higher vaginal depth, were usually closed abdominally as vaginal access was more difficult. Any patient, in need of coincidental minor degrees of vaginal wall repair were better suited for the vaginal vault closure from below.

No stratification was done for patient factors like age, indication, menopausal status, body mass, associated 
pathologies like diabetes, steroids therapy as both groups had fairly similar compositions and far too many confounding minor variables.

The routine follow up was over minimum of 4 months for all; but it has been now nearly 8 years since the initial procedures allowing ample time to present with late complications like vault prolapse.

\section{Results}

The time taken to close only the cuff alone, for method A was 19 min on average (range 8-60) and just 5.5 (range 3-40) minutes when performed vaginally. The exceptional times of 40 minutes in the vaginal closure and the 60 minutes in the abdominal group were outliers from one patient in each group due to difficulty with haemostasis re-encountered during cuff closures and has altered the means to a degree).

As for complications; there was one instance of accidental lower ureteric injury in A and another developed hydro ureter needing stent, but both events seemed unrelated to the step of vault closure as they were most likely injured while sharp dissection of the ureter and the bladder. Therefore, both instances were excluded from the analysis specifically targeted to compare the only step of cuff closure.

Early vault dehiscence occurred subsequently in two patients of A, one was in whom the lower ureteric injury happened. This also resulted later in a large $3 \mathrm{~cm}$ vault granuloma. Too early resumption of sexual activity - before 2 months aided the dehiscence in the other patient in group $\mathrm{A}$, though the exact contribution is uncertain.

There were 2 instances of minor infections of the vault edges in each method, $\mathrm{A}$ and $\mathrm{V}$ not leading to abscess formation.

One case of vault prolapse was encountered after a few months in V.

Two instances of minor degrees of vaginal bleeding went on subsequently to develop vaginal cuff hematomas in the abdominal closure A. Both did not require major re-intervention other than simple application of pressure by packing and drainage by opening the vault.

Complication rates were therefore relatively much higher with the abdominal closure-6 (23\%) instances directly attributable to surgery on the vault; but only 3 (8.3\%) in vaginal closure. The sample size is inadequate to apply complex statistical analysis of these events as with all series $4,7,9,10$ as no single centre could practically perform these newer procedures in very large numbers over a few years unless data from multicentre trials are analysed.

\section{Discussion}

How best to close the vagina at TLH has been debated by many gynaecologists $1,2,4,5,7$ world over, ever since the procedure gained wider acceptance.

Abdominal laparoscopic closure allowed better visualization under magnification. This had both good and bad effects; the bad by catching too little of the vaginal cut edge often made to look bigger under magnification, but actually dissected and caught to a lesser extent. This has been the experience of a few other surgeons too ${ }^{3,4,6,10}$

The infection rates expected logically to be more with vaginal approach hardly differed. Prophylactic and post-operative antibiotic cover probably contributed to minimize differences.

The vaginal manual closure on the other hand allowed a 'bigger solid bite' by the use of a larger needle and a thicker thread. No magnification allowed real size and thickness to be gauged accurately. Better tension in tying knots and approximating tissues with a more real feel to knots with tactile feedback was an advantage. Less expertise is needed as anyone performing a vaginal hysterectomy should be competent in this step.

But the most obvious advantage was the lesser time consumed and the lower complication rates even allowing for chance in the relatively small sample.

\section{Conclusion}

Stronger closure of vault allowing use of a larger needle and thicker bite of vaginal edge may have reduced dehiscence when the cuff is closed vaginally. Authors who do not favour vaginal cuff closure technique at TLH, state difficulty in reaching the cuff and placing sutures especially for women with narrow vaginas. Sri Lankan surgeons who are usually very adept at vaginal surgeries and hysterectomies having performed them in relatively larger numbers would hardly find 
this to be a problem. Sri Lankan patients are of much lesser BMI than those of the West. The big fear of possible contamination by vaginal surgery was not borne out in this study with equal amounts of sepsis between the groups.

It's therefore safe to conclude that cuff closure by vaginal route is both faster and less prone to complications ${ }^{8}$ especially in the initial training of postgraduates introduced to TLH.

\section{Author declarations}

Finanicial assistance: No financial assistance obtained as the research was on procedures performed routinely with minimal expenditure in auditing results.

Conflicts of interest: No conflicts of interest.

\section{Acknowledgements}

The author thanks the registrars and nursing staff who assisted the surgeries and follow up.

\section{References}

1. Hur HC, Guido RS, Mansuria SM, Hacker MR, Sanfilippo JS, Lee TT. Incidence and patient characteristics of vaginal cuff dehiscence after different modes of hysterectomies. J Minim Invasive Gynecol. 2007; 14: 311-17.

2. Iaco PD, Ceccaroni M, Alboni C, Roset B, Sansovini M, D’Alessandro L, et al. Transvaginal evisceration after hysterectomy: is vaginal cuff closure associated with a reduced risk? Eur J Obstet Gynecol Reprod Biol. 2006; 125: 134-38.

3. Cronin B, Sung VW, Matteson KA. Vaginal cuff dehiscence: risk factors and management. Am J Obstet Gynecol. 2012; 206: 284-88.

4. Hwang JH, Lee JK, Lee NW, Lee KW. Vaginal cuff closure: a comparison between the vaginal route and laparoscopic suture in patients undergoing total laparoscopic hysterectomy. Gynecol Obstet Invest. 2011; 71(3): 163-69.

5. Hur HC, Donnellan N, Mansuria S, Barber RE, Guido R, Lee T. Vaginal cuff dehiscence after different modes of hysterectomy. Obstetrics Gynecol. 2011; 118: 794-801.

6. Uccella S, Ghezzi F, Mariani A, Cromi A, Bogani G, Serati M, et al. Vaginal cuff closure after minimally invasive hysterectomy: our experience and systematic review of the literature. Am J Obstet Gynecol. 2011; 205: 119.e1.

7. Siedhoff MT, Yunker AC, Steege JF. Decreased incidence of vaginal cuff dehiscence after laparoscopic closure with bidirectional barbed suture. J Minim Invasive Gynecol. 2011; 18: 218-23.

8. Sunyecz JA. Vaginal cuff closure during total laparoscopic hysterectomy: A new technique has been performed 100 times with very few complications. Am J Obstet Gynecol. 2013; 209: 71.e1-2

9. Blikkendaal MD, Twijnstra AR, Pacquee SC, Rhemrev JP, Smeets MJ, de Kroon CD, et al. Vaginal cuff dehiscence in laparoscopic hysterectomy: influence of various suturing methods of the vaginal vault. Gynecol Surg. 2012; 9: 393-400.

10. Uccella S, Ceccaroni M, Cromi A, Malzoni M, Berretta R, De Iaco P, et al. Vaginal cuff dehiscence in a series of 12,398 hysterectomies: effect of different types of colpotomy and vaginal closure. Obstet Gynecol. 2012; 120: 516. 